\title{
AVAILABILITY IMPROVEMENT OF WDM SYSTEM USING RING STRUCTURES
}

\author{
Ivan RADOŠ ${ }^{*}$
}

\begin{abstract}
This work deals with modelling and availability analysis for the different systems, which are based on WDM (Wavelength Division Multiplying) technology. In the introduction the reasons are stated because of which the high availability of the system is important. Furthermore, the basic terms are introduced pertaining to the availability with particular review of the parallel structure. Then the availability of the system of the various WDM systems is analyzed: point-point, chain and ring with $1+1$ protection of the wave channel as well as the influence of the availability of the nodes and links on the total availability of the analyzed systems. The data on defects intensity and medium repair time of certain components were taken from literature. We assumed in the analysis that it is about WDM system with 16 wave lengths and with $2.5 \mathrm{~Gb} / \mathrm{s}$ as a basic capacity carried by the wave channel. At the end of paper the results are presented of the calculation and comparisons of the availability of the various WDM systems as well as a proposal for improvement of its availability.
\end{abstract}

K e y w or d s: availability, protection, wavelength channel

\section{INTRODUCTION}

Growth of data transmission, as well as the development of the internet traffic, requires transmission systems of huge capacities. Owing to the development of the photonic technologies the systems based on the multiplexing of the wave lengths are developed (Wavelength Division Multiplexing-WDM) which has high transmission speeds through one fiber. In such networks, interruption of the service for any reason, defect at the equipment or human factor, can cause the isolation in the telecommunication terms and huge losses to the customers and network operators. Therefore, the application of the protection in such systems has an extremely significant role for the network operators.

The possibility to guarantee a high availability is the key for the operators in order to keep the existing and attract the new customers, and that mainly reflects on the price of their network [1]. Far more acceptable way of guaranteeing high availability is the use of the various protection strategies. Although there are several protection strategies in this paper we will focus on $1+1$ protection of wave channel which is very similar to protection of the path in the SDH technology [2]. In this paper we will first analyze the availability between two terminals of the WDM systems without protection (point- point, chain) and later the availability of the WDM ring which uses $1+1$ protection of the wave channel.

\section{ON AVAILABILITY IN GENERAL}

The availability of some system $A$ is probability that the system will be functional in the given time, ie that is ratio of time in which the system is correct compared to the total time. Unavailability $U$ is a probability which is complementary to the availability. When reporting on system performances, unavailability is frequently also expressed as a medium failure time MDT (Mean Down Time) in minutes per year

$$
M D T=\frac{M T T R}{M T T R+M T T F}
$$

where the MTTF (Mean Time To Failure) is a medium failure time, and MTTR (Mean Time To Repair) is medium repair time [3]. In this paper we assume that the MTTF of the wave channel is constant regardless of a component age. $M T T F=1 / \lambda$, where $\lambda$ is intensity of the failure which is usually expressed in FIT (Failure In Time-failure in time, $1 \mathrm{FIT}=1$ failure in $10^{9}$ hours).

Availability of the serial structure is equal to the product of the availability of individual elements,

$$
A_{s}=\prod_{i=1}^{n} A_{i} .
$$

Such a structure does not have a possibility to renew the communication between two nodes in case of failure at any element, ie the connection is functional if and only if all elements of the structure are correct.

The significant improvement to the availability can be achieved by applying a parallel structure which means that besides the working path there is a protective one. Of course it is important that these two roads are completely independent $i e$ they do not have common elements [4]. If we mark the availabilities of the working and the protective paths as two completely independent events $(w$ and $p$ ), then the availability of such a structure is a union of two non-disjunctive events, $i e$ events which do not exclude each other mutually because complementary variables do not exist.

$$
A_{p}=P(w \cup p)
$$

* Department of Transmission Systems of the HT Mostar (Croatian Telecommunication), Bosnia and Herzegovina, Ivan.Rados@hteronet.ba 


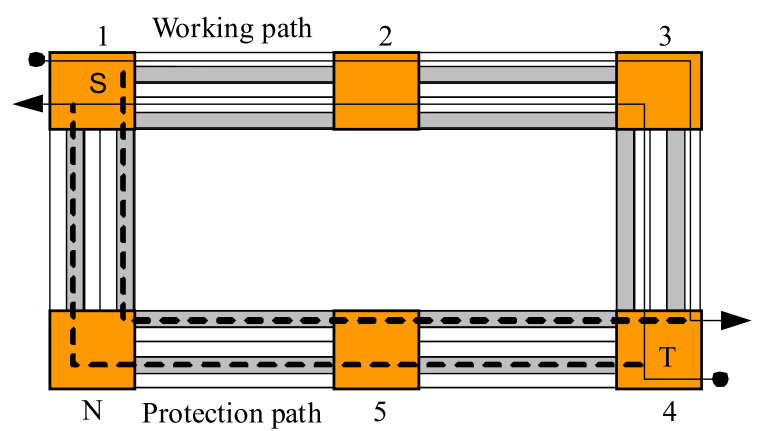

Fig. 1. 1+1 wavelength channel protection under conditions without a failure

For these events to be able to exclude each other mutually it is necessary to turn this union of non-disjunctive events into a union of disjunctive events in which complementary variables appear.

By conducting a simple analysis using De Morgan laws, one finds that the availability is, [5]

$$
A=P\lfloor w \cup(\bar{w} \cap p)\rfloor .
$$

By using the law of distribution bearing in mind the union and intersection

$$
A \cup(B \cap C)=(A \cup B) \cap(\mid \cup C)
$$

and according to the identity $1 \cap p=p$, we get

$$
A_{p}=P(w \cup p)
$$

And this is in fact the availability of the parallel structure. The availability of the connection for some wave channel is $k$

$$
A_{p}=A_{k}=A_{w}+A_{p}-A_{w} A_{p} .
$$

Connection between two nodes with in case of $1+1$ protection will be improper if and only if there is a failure on the working and protection paths. Of course that the connection cannot be set up if a failure occurs on end nodes because they are common elements for the working and protection paths.

\section{ANALYSIS OF AVAILABILITY OF DIFFERENT WDM SYSTEMS}

In this part we will first analyze the availability of WDM system point-point, chain (systems are without redundancy) and WDM ring with $1+1$ protection wave channel.

A network generally consists of $N$ nodes and $N$ links which connect those nodes [6]. In order to define the availability of the wave channel between two nodes we will introduce terms which refer to the availability of the optical link which connects the two nodes.

- $a_{O L_{i}}$, availability of $i$-th link on working path,

- $a_{n_{j}}$, availability of $j$-the node which belongs to the working path.

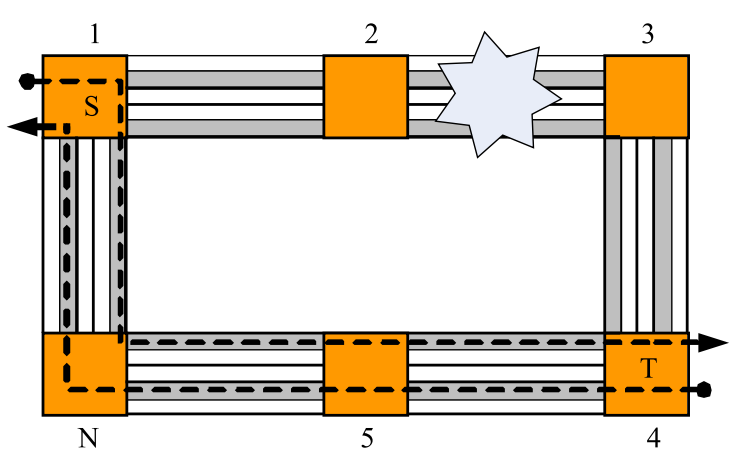

Fig. 2. $1+1$ protection of the wavelength channel in case of failure on the working path

An optical link consists of an optical fibre and an optical amplifier $\left(a_{O A}\right)$. In order for the connection to be functional the optical fibre and amplifier must work properly. The most frequent cause of failure of the optical link is cut optical fibre. Since, when cutting all the fibres in the cable are cut, instead of availability of the fibre the availability of the cable $\left(a_{C A}\right)$ is regarded since failures of fibre and cable are completely mutually dependant.

$$
a_{O L_{i}}=a_{C A} \times a_{O A} .
$$

Nodes in which the wave channel is added/dropped are called "termination nodes. The nodes through which wave channel passes from "west to the "east side and which are located between end nodes are called "passing nodes. Since the wave channel passes through different components within these two types of nodes their availability is different.

$-a_{n_{j} t}$, termination node is working,

- $a_{n_{j} p}$, passing node is working.

If the all nodes of the same type are the same, we have

$$
\begin{aligned}
& a_{n_{j} t}=a_{n t}, \forall j, \\
& a_{n_{j} p}=a_{n p}, \forall j .
\end{aligned}
$$

\subsection{The Availability of WDM Point to Point Systems}

Since the installation of a number of SDH line systems between two nodes is very expensive because the capacities of the fibre optics cables are significantly consumed, a need arose for systems of high transmission capacity which need only two fibres. Such are WDM systems which are based on the multiplexing of wave lengths. First phase in the introduction of the WDM is point-point system. With such systems only end nodes are WDM nodes and between them the optical amplifiers are placed at certain distances. In termination nodes wave channel passes through transmitter $(T X)$, multiplexer $(M U X)$, demultiplexor $(D M U X)$, optical amplifier of power $(B O A)$, preamplifier $(P O A)$ and receiver $(R X)$ so that the availability of the node is equal to the product availability of certain elements [7], 
$a_{n t}=a_{T X} \times a_{M U X} \times a_{D M U X} \times a_{B O A} \times a_{P O A} \times a_{R X}$.

Optical link consists of the optical cable and $m$ optical amplifiers so that its availability is naturally equal to the product of the availability of cable and optical amplifiers

$$
a_{O L_{i}}=a_{C A} \times \prod_{i=1}^{m} a_{O A_{i}} .
$$

Total availability of the point-point system is equal to the product of the availability of the nodes and optical link

$$
\begin{array}{r}
a_{s t}(P)=\prod_{i, j \in P} a_{O L_{i}} \times a_{n_{j}}=\prod_{i \in P} a_{O L_{i}} \times \prod_{j=1}^{2} a_{n_{j}} \\
=a_{n t}^{2} \times \prod_{i \in P} a_{O L_{i}} .
\end{array}
$$

\subsection{The Availability of the WDM Chain}

In the termination nodes wave channel passes through transmitter, multiplexer, demultiplexor, optical power amplifier, optical preamplifier and receiver so that the availability of end node is the same as with the pointpoint system.

In the passing nodes wave channel wave channel passes through optical preamplifier, demultiplexor, multiplexer and optical power amplifier so that the availability of the passing nodes is

$$
a_{n p}=a_{M U X} \times a_{D M U X} \times a_{B O A} \times a_{P O A} .
$$

A failure at any of the components within the nodes as well as in the optical link leads to interruption of communication between the end nodes.

If the wave channel in the unredundant structure passes $m$ optical links and nodes, availability of the communication between the end nodes in such a structure is equal to the product of the availability of the optical links and nodes[8],

$$
\begin{array}{r}
a_{s t}(P)=\prod_{i, j \in P} a_{O L_{i}} \times a_{n_{j}}=\prod_{i \in P} a_{O L_{i}} \times \prod_{j \in P} a_{n_{j}}= \\
a_{n t}^{2} \times a_{n p}^{m-1} \times \prod_{i \in P} a_{O L_{i}} .
\end{array}
$$

It is important to stress here that the optical link consist of optical cable only.

\subsection{Availability of WDM Ring with $1+1$ Protec- tion of Wavelength Channel}

In the ring network which uses $1+1$ protection of the wave channel, wave channel in the source node is duplicated and is transmitted simultaneously in the both directions of the ring, working and protection directions.
Under normal circumstances, in the end node, receiver receives two copies of the signal (with different delay) and chooses the better one.

In case of failure on the working path receiver chooses the signal which it receives from the protection path. It is important here that working and protection paths do not have common elements, so that one failure would not cause total interruption of the communication, and that means that the failures of the elements on the working and protection paths are completely independent. For each wave channel of the working path the appropriate wave channel at the protection path is booked, and therefore we speak about $1+1$ protection of the wave channel. Wave channel in the end nodes passes through transmitter, splitter, multiplexer, optical power amplifier, optical preamplifier, demultiplexor, switch and receiver so that the availability of the end nodes is

$$
\begin{array}{r}
a_{n t}=a_{T X} \times a_{S P L} \times a_{M U X} \times a_{D M U X} \times a_{B O A} \times a_{P O A} \times \\
a_{S W} \times a_{R X} .
\end{array}
$$

Availability of the passing nodes is the same as with WDM chain because wave channel passes the same components. If the wave channel at the working path $P_{0}$ passes $m$ optical links between end nodes, availability of the working path is equal to the product of the availability of the optical links and nodes through which wave channel passes, [8]

$$
\begin{array}{r}
a_{s t}\left(P_{0}\right)=\prod_{i, j \in P_{0}} a_{O L_{i}} \times a_{n_{j}}=\prod_{i \in P_{0}} a_{O L_{i}} \times \prod_{j \in P_{0}} a_{n_{j}}= \\
a_{n t}^{2} \times a_{n p}^{m-1} \times \prod_{i=P_{0}}^{m} a_{O L_{i}} .
\end{array}
$$

In case of failure on the working path, wave channel passes $N-m$ optical links and $N-m-1$ nodes at the protective path $P_{1}$.

Availability of the protection path is, [10]

$$
\begin{array}{r}
a_{s t}=\prod_{i, j \in P_{1}} a_{O L_{i}} \times a_{n_{j}}=\prod_{i \in P_{1}} a_{O L_{i}} \times \prod_{j \in P_{1}} a_{n_{j}}= \\
a_{n t}^{2} \times a_{n p}^{N-m-1} \times \prod i \in P_{1}^{N-m} a_{O L_{i}} .
\end{array}
$$

Availability of the wave channel between $s$ and $t$ nodes is completely defined with these two paths, so the availability of the wave channel in the case of $1+1$ protection is calculated as the availability of the parallel structure $A_{s t}$ composed of two branches whose failures are completely independent.

$$
A_{s t}(a)=a_{s t}\left(P_{0}\right)+a_{s t}\left(P_{1}\right)-\left[a_{s t}\left(P_{0}\right) \times a_{s t}\left(P_{1}\right),\right.
$$

$$
\begin{aligned}
& A_{s t}(a)=a_{n t}^{2} \times a_{n p}^{m-1} \times \prod_{i \in P_{0}}^{m} a_{O L_{i}}+a_{n t}^{2} \times a_{n p}^{N-m-1} \times \prod_{i \in P_{1}}^{N-m} a_{O L_{i}} \\
& -\left\{a_{n t}^{2} \times a_{n p}^{N-m-1} \times \prod_{i \in P_{0}}^{m} a_{O L_{i}} a_{n t}^{2} \times a_{n p}^{N-m-1} \times \prod_{i \in P_{1}}^{N-m} a_{O L_{i}}\right\}
\end{aligned}
$$


where $a$ marks availability of optical links and nodes.

Although in the in the equation's large brackets we have a product of two equal members $a_{n t}^{2} \times a_{n t}^{2}$, only one is taken in the expression for availability $a_{n t}^{2}$ because the cause of the failure of the node is the same so we have

$$
\begin{gathered}
A_{s t}(a)=a_{n t}^{2} \times\left\{a_{n p}^{m-1} \times \prod_{i \in P_{0}}^{m} a_{O L_{i}}+a_{n p}^{N-m-1} \times \prod_{i \in P_{1}}^{N-m} a_{O L_{i}}\right. \\
\left.\quad-a_{n p}^{N-2} \times \prod_{i \in P_{0}, P_{1}}^{N} a_{O L_{i}}\right\} .
\end{gathered}
$$

\section{ANALYSIS OF RESULTS}

In order to calculate the availability of the WDM system it is necessary to know the availability of the nodes and optical links. For calculation of the availability in general it is necessary to know the intensity of the failures of certain elements as well as the time of their repair. These data are taken from the various literature and previously published papers [9].

As it can be seen in the Tables 1, 2 and 3 the decrease of the time for repair both of the cable and the device significantly decreases medium outage time and that by all means reflects on the availability of the entire system.

We will carry out the analysis of the availability of analysed systems with the assumption that the systems comprise 8 and 12 nodes, 16 wave lengths, 560 and $960 \mathrm{~km}$ length of the optical links and $80 \mathrm{~km}$ distance between optical amplifiers ie nodes. We will also analyse the influence of the decrease in the cable repair time and device on the system availability.

As it can be seen from the above Tables 4-7, the pointpoint system has the highest unavailability. Although the chain system has WDM nodes in places of optical amplifiers, its unavailability is somewhat smaller than that of the point-point system because of the very high failure intensity of optical amplifiers [9].

Table 1. Failure intensity, unavailability and MDT for optical link $(\mathrm{MTTR}=21$ and $15 \mathrm{~h})$

\begin{tabular}{ccc}
\hline$\lambda(\mathrm{FIT} / \mathrm{km})$ & $U \times 10^{-6}$ & $M D T(\mathrm{~min} /$ year $)$ \\
\hline 114 & 2.394 & 1.26 \\
114 & 1.710 & 0.90 \\
\hline
\end{tabular}

Table 2. Unavailability and MDT for nodes for the system point to point

\begin{tabular}{lcc}
\hline$M T T R=6 \mathrm{~h}$ & $U \times 10^{-5}$ & $M D T(\mathrm{~min} /$ year $)$ \\
\hline Termination & 5.759 & 30.27 \\
Passing & 4.319 & 22.71 \\
\hline$M T T R=4 \mathrm{~h}$ & & \\
\hline Termination & 3.840 & 20.18 \\
Passing & 2.880 & 15.14 \\
\hline
\end{tabular}

Table 3. Unavailability and MDT for nodes of WDM ring with $1+1$ protection of the wave channel

\begin{tabular}{lcc}
\hline$M T T R=6 \mathrm{~h}$ & $U \times 10^{-5}$ & $M D T(\mathrm{~min} /$ year $)$ \\
\hline Termination & 4.049 & 21.29 \\
Passing & 4.319 & 22.71 \\
\hline$M T T R=4 \mathrm{~h}$ & & \\
\hline Termination & 2.700 & 14.19 \\
Passing & 2.880 & 15.14 \\
\hline
\end{tabular}

Table 4. Unavailability and MDT for various systems $(N=$ 8 nodes)

\begin{tabular}{ccc}
\hline$M T T R=21$ and $6 \mathrm{~h}$ & $U \times 10^{-5}$ & $M D T(\min /$ year $)$ \\
\hline Point-point & 191.82 & 1008.51 \\
Chain & 171.31 & 900.48 \\
Ring $(1+1)$ & 8.16 & 42.91 \\
\hline MTTR=15 and 4h & & \\
\hline Point-point & 136.54 & 717.83 \\
Chain & 120.64 & 634.02 \\
Ring $(1+1)$ & 5.43 & 28.55 \\
\hline
\end{tabular}

Table 5. Unavailability and MDT for various systems $(N=$ 8 nodes)

\begin{tabular}{ccc}
\hline$M T T R=21$ and $4 \mathrm{~h}$ & $U \times 10^{-5}$ & $M D T(\mathrm{~min} /$ year $)$ \\
\hline Point-point & 188.02 & 988.33 \\
Chain & 158.86 & 834.89 \\
Ring $(1+1)$ & 5.45 & 28.68 \\
\hline$M T T R=15$ and $6 \mathrm{~h}$ & & \\
\hline Point-point & 140.45 & 738.01 \\
Chain & 133.12 & 699.61 \\
Ring $(1+1)$ & 8.13 & 42.77 \\
\hline
\end{tabular}

Table 6. Unavailability and MDT for various systems ( $N=$ 12 nodes)

\begin{tabular}{ccc}
\hline$M T T R=21$ and $4 \mathrm{~h}$ & $U \times 10^{-5}$ & $M D T(\min /$ year $)$ \\
\hline Point-point & 314.27 & 1651.76 \\
Chain & 265.78 & 1396.92 \\
Ring $(1+1)$ & 556.64 & 29.26 \\
\hline$M T T R=15$ and $6 \mathrm{~h}$ & & \\
\hline Point-point & 230.75 & 1212.55 \\
Chain & 218.65 & 1149.01 \\
Ring $(1+1)$ & 8.26 & 43.14 \\
\hline
\end{tabular}

Table 7. Unavailability and MDT for various systems ( $N=$ 12 nodes)

\begin{tabular}{ccc}
\hline$M T T R=21$ and $6 \mathrm{~h}$ & $U \times 10^{-5}$ & $M D T(\min /$ year $)$ \\
\hline Point-point & 318.13 & 1671.94 \\
Chain & 284.05 & 1492.79 \\
Ring $(1+1)$ & 8.28 & 43.55 \\
\hline$M T T R=15$ and $4 \mathrm{~h}$ & & \\
\hline Point-point & 226.86 & 1192.37 \\
Chain & 200.32 & 1053.15 \\
Ring $(1+1)$ & 5.49 & 28.87
\end{tabular}


Naturally the WDM ring with protection of the wave channel has the smallest unavailability (35 times smaller than the one of the point -point and 31 time than that of the chain) which is understandable because it is about the parallel structure at which the failures of the certain elements are completely independent. Decrease of repair time of the cable from 21 to 15 hours significantly decreases the unavailability with the system point-point (around $27 \%$ ) and chain (around 22\%) and insignificantly with WDM ring (less than 1\%). The reason is in the fact that the influence of the links on the total unavailability in the point-point system is around $95 \%$, chain around $80 \%$ and ring with $1+1$ protection of the wave channel between 0.5 and $1 \%$.

Decrease of the repair time of the device from 6 to 4 hour has the highest influence on the decrease of the unavailability with the ring, around $23 \%$, chain around $7 \%$ and point-point by around $2 \%$. Result of such influence is in the fact that significantly higher influence of the nodes on the total unavailability with the ring structure (between 98 and $99 \%$ ) than with the chain (around 20\%) and point-point (around $2 \%$ ) and therefore it is understandable that the decrease of the device repair time has exactly such influence. Of course, in the WDM ring end nodes have the highest influence on the total unavailability because the failure on them leads to interruption of communication between two nodes.

End nodes have influence on the unavailability of both the point-point system and chain system but the influence is smaller due to significantly higher influence of the cable on the total unavailability. The smallest unavailability of the system is obtained by decreasing the repair time of both the cable (from 21 to 15) and the device (from 6 to 4 hours) and, expressed in percentages, it ranges around $30 \%$ for point-point, $29 \%$ for chain and $33 \%$ for ring. As it can be seen, the difference in the total decrease of the unavailability is not big because in certain cases the influence of the cable is dominant and in other influence of nodes so almost the same decrease of the unavailability is obtained in case of decrease of repair time of cables and nodes.

\section{CONCLUSION}

The analysis of the results in this paper shows that the point-point system has the least availability. The significant improvement of the availability is achieved by application of the WDM ring which uses $1+1$ protection of the wave channel because it is actually about parallel structure whose failures are completely independent. Only in case of the failure on both sides of the ring and the end nodes does the system become unavailable.

Point-point and chain systems have several times less availability because they in fact have a serial structure in which failure at any of the components leads to an interruption of communication between two nodes. It is also noticeable that, in general, the availability of different WDM systems is decreased with an increase in the length of the optical link and number of nodes, in particular for unredundant systems.

Significant improvement of the availability with all WDM systems can be achieved by decreasing the repair time both of the optical links and the devices and that means with better organization of the maintenance teams. The conclusion is that we need to go for building of ring systems because in that way the users can be offered high availability of services.

\section{REFERENCES}

[1] WILlEMS, G.-ARIJS, P.-PARYS, V. W.-DEMEESTER, P.: Capacity vs Availability Trade-offs in Mesh-Restorable WDM Networks, Design of Reliable Communication Networks (DRCN), Proc., Oct 01, Budapest, Hungary.

[2] SCHUPKE, A. D.: Reliability Models of WDM Self-Healing Rings, Second International Workshop on the Design of Reliable Communication Networks (DRCN), Munich, Germany, April 9-12, 2000

[3] JURDANA, I.-MIKAC, B.: An Availability Analysis of Optical Cables, Proceedings WAON`98, pp. 153-160.

[4] INKRET, R.: Shaping of Entirely Optical Transmission Network with Wave Multiplex, Masters degree thesis, Faculty of Electrical engineering and computing/IT; University in Zagreb, 1998.

[5] INKRET, R.: Availability Modelling of Multi-Service All-Optical Transmission Network, Doctoral Thesis, University of Zagreb, Faculty of Electrical Engineering and Computing, Zagreb, 2004.

[6] ARCi, D.-MAiER, G.-PATtAvinA, A.-PETECCHI, D. -TORNATORE, M. : Availability Models for Protection Techniques in WDM Networks, Design of Reliable Communication Networks (DRCN) (Oct 2003), 158-166, Banff, Alberta.

[7] INKRET, R.-MIKAC, B.: Availability Analysis of Different WDM Network Protection Scenarios, Proceedings WAON`98, pp. 121-128.

[8] RADOS, I.: Influence of common Path on Availability of the Ring Network, Journal of Telecommunication and Information Technology No. .3 (2004), National Institute of Telecommunication, Poland.

[9] TRSTEnsky, D.-SCHWARTZ, L.-TRUnKVALTER, M. -BAROŠ, R.: Reliability of Telecommunication System by Various Repair Regime: International Conference ELEKTRO 2004, Advances in Electrical and Electronic Engineering 3/2004 No. 2, 25-26, ŽU Žilina.

Recdeived 10 September 2010

Ivan RADO ̌ received the BSc degree in electrical engineering from the University of Split, Croatia, in 1983, and MSc degree from the University of Zagreb, in 2000. In 1985 he joined the PTT (Post and Telecommunication) Tomislavgrad. Since 1992 he works at Department of Transmission Systems of the HT Mostar (Croatian Telecommunication). His research interests include: digital transmission systems, optical systems and networks, availability and reliability of telecommunication systems. He has published 10 papers in international conference proceedings and 5 papers in international journals. 